\title{
EVALUACIÓN DE CALIDAD DE RESÚMENES DE TESIS DE UN PROGRAMA DE MAGÍSTER EN ENFERMERIA
}

\author{
QUALITY ASSESSMENT OF THESES ABSTRACTS FROM A \\ NURSING MASTER'S DEGREE PROGRAM
}

\author{
Tatiana Paravic Klijn ${ }^{*}$ \\ Mónica Burgos Moreno**
}

\begin{abstract}
RESUMEN
El artículo presenta un estudio de tipo cuantitativo, descriptivo, correlacional, retrospectivo, cuyo objetivo es la evaluación de calidad de resúmenes de tesis de un programa de magíster en enfermería en una universidad chilena. La población en estudio quedó constituida por 84 resúmenes disponibles desde el período comprendido entre 1983-2007. El instrumento recolector de datos utilizado fue la pauta de evaluación de artículos científicos elaborada por Bobenrieth (2002) en las dimensiones de: título, identificación de autores y cuerpo del resumen, las que fueron modificadas para esta investigación. Los resultados muestran que el $85,7 \%$ de los autores de los resúmenes corresponden a enfermeras del mundo académico y que el abordaje metodológico de los estudios es cuantitativo en un $92,9 \%$. Se constató que ninguno de los resúmenes evaluados logró el máximo puntaje en la escala. Las principales deficiencias detectadas son en la extensión de las palabras del título, la identificación incompleta de los autores y las conclusiones no relacionadas con objetivos ni resultados planteados. Resultó estadísticamente significativa la correlación de año de ingreso del autor y puntaje obtenido en la evaluación de calidad de los resúmenes de tesis $(\mathrm{p}=0,03)$. Llama la atención la gran dispersión de fenómenos estudiados. Se concluye que a año más reciente de ingreso del alumno al programa, mayores puntajes en la calidad de los resúmenes de las tesis. Existen aspectos dentro de las diferentes dimensiones evaluadas que requieren ser mejorados para elevar la calidad de la producción científica del postgrado.
\end{abstract}

Palabras clave: Evaluación, calidad, resumen tesis, magíster en enfermería.

\begin{abstract}
This paper presents a quantitative, descriptive, correlational and retrospective study whose purpose is to assess the quality of theses abstracts from a Nursing Master's Degree Program in a Chilean university. The study considers 84 abstracts available since 1983-2007. The data was collected for this study using the assessment instrument for scientific articles proposed by Bobenrieth (2002) that considers: title, identification of authors, and the abstract, which were modified for this study. The results show that $85.7 \%$ of the abstract authors are nurses working in the academic world and that the methodological approach of the studies is $92.9 \%$ quantitative. We found that none of the abstracts evaluated obtained the maximum score. The main deficiencies detected were the number of words in the titles, incomplete author data, and the conclusions, which were unrelated either to the objectives or the results proposed. The correlation between the author's year of entry into the program and the score obtained in the quality assessment of the theses abstracts was statistically significant $(\mathrm{p}=0.03)$. We can conclude that the more recent the year of entry of the student into the program, the higher the scores in the quality of the abstracts. Certain aspects of the different dimensions evaluated need to be improved in order to increase the quality in the scientific production of the postgraduate program.
\end{abstract}

Key words: Assessment/evaluation, quality, thesis abstract, nursing master degree.

Fecha recepción: 11/12/08 Fecha aceptación: 13/08/09

\footnotetext{
* Doctora en Enfermería. Magíster en Enfermería en Salud Comunitaria. Profesora Titular, Departamento de Enfermería. Universidad de Concepción. Direc: Roosevelt esquina Janequeo. Concepción, Chile. Fono: (56)(41)2204449. E-mail: tparavic@udec.cl

${ }^{* *}$ Doctora (C) en Enfermería. Magíster en Enfermería. Profesora Asistente, Departamento de Enfermería. Universidad de Concepción.E-mail: moniburgos@udec.cl
} 


\section{INTRODUCCIÓN}

En la sociedad del conocimiento en la que hoy vivimos, la evaluación de la calidad ha trascendido también a la producción científica, representada por las tesis de grado, investigaciones y publicaciones científicas, entre otras.

La evaluación de la producción científica representa un elemento clave en la toma de decisiones, que han de propiciar la mejora de una institución o programa evaluado, contribuyendo así en su transformación. La evaluación sirve para promover espacios permanentes de reflexión y profundización teórica en el área investigativa, y debe ser considerada como un permanente proceso formativo. El proceso de evaluación representa en sí mismo un control de calidad acerca de cómo se está realizando la investigación (1).

Ahora bien, hablar de evaluación de la calidad de las investigaciones implica la identificación de las fortalezas y debilidades del informe de una investigación, es decir, realizar una lectura crítica de su informe. Esto incluye el evaluar los aspectos del estudio para juzgar sus méritos, significados, alcances y limitaciones. Durante muchos años el proceso de escritura de informes de investigación fue más bien intuitivo, autoformativo y desestructurado para los científicos. Sin embargo, en el siglo XIX fueron escritos los primeros informes con estructura similar a los actuales. Posteriormente fueron las propias revistas, en el ámbito de las ciencias de la salud, quienes consolidando su prestigio establecieron normas específicas para la presentación y posterior publicación de investigaciones científicas (2).

Bobenrieth (3) establece que para evaluar la calidad de una investigación el lector debe conocer el proceso, las etapas, terminologías, fundamentos de la investigación científica y contar con pautas de lectura crítica a seguir en cada capítulo del trabajo. Disponer de una pauta, señala este autor, sirve como nor- ma o modelo estándar sobre el "deber ser" del proceso de escritura de una investigación científica que se precie como tal.

El interés actual por la evaluación de la investigación -en sentido general- se ha extendido ampliamente. Por un lado, es un tema que continúa atrayendo la atención de los gobiernos y de las agencias de financiación; por otro, el interés de la propia comunidad científica sobre los procesos de evaluación principalmente en las revistas científicas. Sin duda el carácter dinámico y competitivo de la ciencia ha ayudado a este creciente interés.

Para garantizar que la investigación sea de máxima calidad, las entidades financiadoras, las revistas y diversas instituciones involucradas en el tema disponen actualmente de herramientas de evaluación tan eficaces como son la bibliometría, la evaluación por pares y diversos métodos para medir el impacto social y económico que tienen las investigaciones.

Hay quienes opinan que la evaluación rigurosa de la calidad de las investigaciones y la renovación de los procesos resultantes debe gestarse en las universidades, iniciándose con un proceso reflexivo y participativo unido por el interés del saber, el conocimiento y la búsqueda de la verdad (4). Esto se debe a que la producción científica ha estado históricamente ligada a las universidades, especialmente a través de programas de postgrado, los que persiguen estimular el desarrollo de la investigación científica a través de una preparación adecuada de investigadores $(5,6)$ y que contribuyan ciertamente al desarrollo del conocimiento en las diferentes disciplinas. De este modo, la academia representa un papel protagónico en la formación de personas en el área investigativa y los programas de postgrado son responsables de la mayor parte de la producción científica (7). Así han surgido distintos postgrados orientados a la formación de investigadores a través principalmente de programas de doctorados y maestrías, que pretenden dar respuesta a la 
necesidad de formar recursos humanos altamente calificados.

Los programas de maestría por una parte profundizan los conocimientos temáticos, y por otra fomentan el desarrollo de capacidades para generar soluciones frente a un problema determinado, el cual es resuelto y explicado en una tesis, que representa el producto final de esta formación (8).

La tradicional tesis de grado está orientada fundamentalmente a la publicación científica, cumpliendo con el requisito del último paso del proceso de investigación que es precisamente la publicación. Entonces es imperativo que los profesores guías de tesis procuren la calidad de los manuscritos (9). En este punto, es importante resaltar que es frecuente que no se realicen evaluaciones periódicas a las tesis, ni a los tutores, ni a los programas, y por ello no son evidenciadas las deficiencias que pueden presentar y como consecuencia no son corregidas (10).

Los programas académicos de postgrado requieren evaluar sus investigaciones para descubrir las orientaciones de sus tendencias actuales, identificar aspectos a mejorar y representan un insumo fundamental para efectuar proyecciones relacionadas con las necesidades futuras de producción científica de las diferentes disciplinas (11).

En las ciencias de la salud diversas disciplinas han realizado investigaciones acerca de la producción científica representada en tesis de postgrado, con el objetivo de evaluar diversos aspectos, entre los que se destacan; cantidad de tesis defendidas, fenómenos de estudio, análisis bibliométrico de citas y el rol del tutor de tesis $(12,13,14,15)$.

Sin embargo, Sanabria y Bullón (16) señalan que los estudios sobre la calidad de las tesis universitarias en el área de salud son muy escasos, especialmente en enfermería, y que es de vital importancia su conocimiento como indicador de la calidad de la formación.

Enfermería como disciplina ha experimentado grandes avances en los últimos años, expresado en el progresivo aumento de los programas de postgrado y de su producción científica (1). Esta producción ha sido objeto de diversos estudios que han analizado el quehacer científico de enfermería a través de investigaciones basadas en tesis, siendo el foco principal los fenómenos de estudio $(18,19,20,21,22)$. A pesar del interés por el estudio de las tesis, la evaluación de la calidad como foco de investigación representa actualmente un terreno poco explorado.

En este contexto, destaca una investigación realizada por Oria et al. (23), quienes analizaron los resúmenes de tesis como indicadores de calidad de las mismos en un programa de Doctorado en Enfermería en Brasil, en cuyos resultados destaca que las tesis defendidas en años recientes al estudio mostraban puntuaciones más altas en sus resúmenes, que aquellas presentadas en años anteriores. Los autores sostienen que éste es un hecho que manifiesta la creciente calidad de las tesis, y por tanto del programa en la que es realizada.

Frente a lo expuesto anteriormente, las autoras del presente estudio consideran que enfermería en la actualidad está llamada a reflexionar y evaluar acerca de la construcción de la calidad del conocimiento elaborado. Esto se traduce en el análisis y evaluación de la producción científica que emerge en los programas de postgrado, las tesis. Surge en este contexto el interés por evaluar la calidad de los resúmenes de tesis de un programa de magíster en enfermería de una universidad chilena, como un indicador de la calidad de las tesis mismas.

\section{OBJETIVO GENERAL}

Evaluar la calidad de los resúmenes de tesis de un programa de magíster en enfermería de una universidad, durante los años 19832007. 


\section{OBJETIVOS ESPECÍFICOS}

- Caracterizar el perfil de los autores según sexo, actividad y años de estudio en el programa.

- Identificar los fenómenos estudiados.

- Identificar los sujetos de estudio.

- Identificar los abordajes metodológicos utilizados.

- Medir la calidad global de los resúmenes.

- Medir la calidad de las diversas dimensiones que componen el resumen; título, autor y cuerpo del resumen

- Relacionar los años de ingreso al programa de magíster con puntajes obtenidos en las diversas dimensiones del resumen.

- Comparar la actividad de los autores con los puntajes obtenidos

\section{HIPÓTESIS OPERACIONALES}

1. A años más recientes de ingreso al programa de magíster, mayor puntaje de evaluación de la calidad global de los resúmenes de tesis.

2. A años más recientes de ingreso al programa de magíster mayor puntaje de evaluación de calidad en las diversas dimensiones del resumen.

3. Los autores académicos presentan mejores puntajes en la evaluación de la calidad global de los resúmenes de tesis que los autores asistenciales.

4. Los autores académicos presentan mejores puntajes en la evaluación de la calidad de las diversas dimensiones.

\section{MATERIAL Y MÉTODO}

La presente investigación corresponde a un estudio de tipo cuantitativo, descriptivocorrelacional, y retrospectivo. La unidad de análisis fue establecida como: "el resumen de tesis del Programa de Magíster en Enfermería". Del total de 100 tesis aprobadas en este programa durante el período comprendido entre los años 1983 y 2007, fueron incluidas aquellas tesis disponibles en las bibliotecas de la universidad en la que se efectuó la investigación, quedando constituida la población del estudio por 84 resúmenes de tesis, lo que equivale al $84 \%$ de la población.

El Instrumento recolector de datos utilizado estuvo conformado por dos partes. La primera que recoge datos de identificación (año ingreso, año egreso, sexo, actividad del autor y abordaje de la investigación) y la segunda parte corresponde a la pauta de evaluación de artículos científicos en las dimensiones de: título, autores y cuerpo del resumen, elaborada por Bobenrieth en el año 2002 (3) y modificada para esta investigación.

Para el procesamiento estadístico de los datos se utilizó el programa SPSS 15.0. Empleando estadística descriptiva y análisis bivariado, aplicando estadígrafos de t de Student y correlación de Pearson. Se consideró para este estudio como significación estadística valores de $\mathrm{p}<0.05$.

\section{RESULTADOS Y DISCUSIÓN}

\section{Tabla 1}

El cuidado para el mantenimiento y mejora de la vida humana tradicionalmente se ha relacionado como una labor femenina, razón por la cual la enfermería -en el contexto de la educación superior- tradicionalmente ha sido considerada una carrera femenina. Debido a esto el hallazgo de una diferencia porcentual importante entre mujeres y hombres graduados en el período de estudio es esperado.

Respecto a la actividad del autor, la mayoría desempeñaban labores docentes y el resto de tipo asistencial. Las actividades docentes eran realizadas por académicos de diferen- 
tes universidades, y las de tipo asistencial como profesionales clínicos en diferentes establecimientos de salud. En este último ámbito laboral existen menos incentivos en la realización de estudios de postgrado, poco reconocimiento institucional y menos financiero. Sin embargo, la importancia de la investigación en la disciplina en todos los ámbitos es ampliamente reconocida. Esto se relaciona con lo señalado por Mendoza (24), respecto al amplio conocimiento de que la investigación científica es necesaria para contribuir a otorgar cuidados de mayor calidad basados en evidencia científica, pero que frecuentemente las perspectivas de desarrollo en investigación en enfermería pierden prioridad en función de otras áreas de ejercicio de la profesión (asistencia, educación o administración), y que no permiten disponer de tiempo necesario para la labor investigativa. Cuando existe el interés real en basar la práctica en evidencia científica a comprobar, generalmente se realiza en el contexto de experiencias formativas de cursos de postgrado.

Similares resultados se han arrojado en otros estudios, en los que la producción científica de enfermería proviene del área de la academia (17) Esto dice relación con la motivación y requisito directo que tienen las enfermeras docentes para iniciar estudios de postgrado, exigencia que no se presenta en el mundo de la asistencialidad.

\section{Tabla 2}

Es posible observar que el menor porcentaje de los autores concluye sus estudios incluyendo la tesis en el período de dos años. Respecto a este fenómeno, Carlino (25) menciona que los estudiantes de postgrado, por lo general, concluyen las asignaturas programadas, pero no terminan la elaboración de la tesis, pasando a integrar el grupo de los denominados "todo salvo la tesis" (All But Dissertation). Otro problema de los postgrados, relacionado con éste, es el alargamiento de los tiempos de entrega de las tesis. Esto último puede estar asociado a múltiples causas, tales como; falta de reconocimiento en los lugares de trabajo, tiempo de dedicación, motivación con la temática, relación con el tutor o guía, entre otros. Además, se puede considerar pertinente en este punto, mencionar que como la mayoría de los autores en este estudio son mujeres, el género femenino y los múltiples roles sociales asociados a éste, también puede ser un determinante en el tiempo de egreso del programa. Un estudio argentino respecto a programas de doctorado concluye que de las personas que cursan estudios de postgrado en el extranjero, el $20 \%$ de los hombres terminan sus estudios de postgrado en menos de cuatro años, el porcentaje de mujeres en esta misma situación no sobrepasa el 9\% (1).

Valarino (15) en Venezuela, en un estudio censal, encontró que el 35\% de la población de estudiantes de postgrado, habiendo completado los requerimientos de las asignaturas del programa de estudio, no concluyó el trabajo de grado o tesis; es decir, eran estudiantes Todo Menos Tesis (TMT), mientras que sólo el 13\% se graduó después de 9 años. El TMT en los doctorados (40 a 49\%) era el doble que en las maestrías (20 a 21\%), y la deserción fue similar, alrededor de 63 a 67\%.

\section{Tabla 3}

Llama la atención la cantidad de fenómenos en la categoría de "otros fenómenos" que no pudieron ser agrupados en ninguna de las otras categorías establecidas. Esto podría estar indicando la ausencia de líneas de investigación del programa, entendiendo por línea de investigación al conjunto de investigaciones sobre una problemática común desde diferentes enfoques teóricos y metodológicos o la no adhesión a líneas establecidas. Según Velandia (27) en las distintas unidades universitarias comprometidas con el proceso investigativo, ya que es parte de su quehacer académico, deben definir líneas o áreas de investigación $(5,6)$. 


\section{Tabla 4}

Debido a los múltiples sujetos de los estudios, sólo fue posible clasificarlos en individuos, familias y comunidad. Se puede observar la baja producción en familias y comunidad, dando énfasis al individuo y en especial al colectivo de enfermeras; lo que puede interpretarse como un grado de inmadurez que presenta el desarrollo de la disciplina (24).

\section{Tabla 5}

En la tabla destaca que el mayor porcentaje de resúmenes en estudio corresponden al abordaje metodológico cuantitativo. Este hallazgo coincide con diversos estudios que se han realizado en artículos de la disciplina publicados en diversas revistas. Mendoza y Paravic (28) en un estudio de publicaciones de enfermería en Chile destacan que el 93,5\% de los estudios eran abordados bajo el paradigma cuantitativo. Alarcón y Astudillo (17) observaron que el $80,8 \%$ de las investigaciones publicadas en revistas latinoamericanas de enfermería utilizaban el método cuantitativo, el 17,3\% cualitativos y tan sólo un 1,3\% utilizaron un acercamiento mixto.

Aunque, según Amescua y Gálvez (29) los investigadores de salud, médicos, enfermeras, psicólogos, entre otros, han incorporado crecientemente metodologías cualitativas, por cuanto éstas permiten conocer y comprender aspectos de la experiencia humana del otro cuando los análisis basados en las estadísticas son limitados. Las investigaciones en las ciencias de la salud en general siguen estando estrechamente relacionadas a lo cuantitativo, lo que Arteaga (30) asocia a la formación que reciben los investigadores en ciencias de la salud. Esto se relaciona con la frecuente presentación de las ciencias de la salud como un conjunto de disciplinas integradas a las ciencias de la naturaleza, asociadas directamente al modelo biomédico, fuertemente relacionado a metodologías cuantitativas (31).

\section{Tabla 6}

De los resúmenes estudiados, ninguno obtuvo el máximo puntaje global de la escala de 52 puntos. La media obtenida fue de 37,8 puntos, desviándose cada estudio como promedio 5,68 respecto a esta media. Un $75 \%$ de los resúmenes presentó un puntaje inferior a 42 puntos.

Estos datos evidencian que en general, de acuerdo a la pauta aplicada, existen aspectos que deben mejorarse. Los resultados obtenidos en el estudio realizado por Oria et al. (23) arrojaron, de igual forma, aspectos que en los resúmenes deben ser corregidos, concluyendo que un importante porcentaje de resúmenes que habían sido aprobados por el programa de ese estudio eran deficientes.

\section{Tabla 7}

Destaca que sólo un 61,9\% de los resúmenes presenta títulos concisos. Respecto a este punto, los autores Rosell y Bobenrieth (2) señalan que uno de los principales defectos en la redacción de los títulos es precisamente títulos muy largos, con información redundante y confusa. El título representa el primer dato disponible de una investigación, por tanto debe ser expresado en palabras precisas al tema del estudio, ser atractivo $y$ de estructura sencilla.

\section{Tabla 8}

Un gran porcentaje de títulos evaluados obtuvo el máximo de 12 puntos. La media de los puntajes obtenidos es de 11 puntos, desviándose en un punto las evaluaciones del título respecto a esta media.

\section{Tabla 9}

En este estudio los trabajos fueron presentados con la identificación del autor y sólo algunos de ellos, además, con la identificación del tutor y/o del profesor guía. Sin embargo el mayor porcentaje de los trabajos revisados no presentaban el nombre completo o utilizaban abreviaturas no permitidas.

Es imperativo insistir en que la identifi- 
cación de los autores (tesista y guía) es primordial, Rosell y Bobenrieth (2) afirman que esta identificación debe ser clara, sin la utilización de abreviaturas que pueden prestarse para confusión y malos entendidos. Es importante destacar que si bien es cierto el mayor trabajo del proceso investigativo es del candidato/a al grado de magíster, el rol que juega el guía o tutor de tesis es preponderante. Tal ha sido considerada su importancia que ha sido objeto de estudio de investigación; por ejemplo, Rosas et al. (10) llegaron a determinar que la supervisión del trabajo del guía de tesis representaba uno de los factores fundamentales en el desarrollo y término del proceso investigativo.

\section{Tabla 10}

Se obtuvo un promedio de 2,89 puntos para esta dimensión, desviándose en casi 1 punto de la media. El rango en que se movieron estos valores fue de 2 a 4 .

\section{Tabla 11}

Dentro de la dimensión evaluación de la calidad del cuerpo del resumen los ítemes relacionados con las conclusiones fueron los que presentaron mayores deficiencias, en este sentido, los resúmenes no presentaban conclusiones relacionadas con los objetivos y/o resultados, presentando frecuentemente muchas sugerencias y repitiendo resultados presentados con anterioridad como conclusiones de la investigación.

Un 35,7 \% de los resúmenes presenta descriptores relacionados directamente con el título, un menor porcentaje de los trabajos expuso términos no relacionados en su totalidad con el título de la investigación y un alto porcentaje omitió los descriptores.

\section{Tabla 12}

De los cuerpos de resúmenes de tesis estudiados, cabe destacar que ninguno de ellos obtuvo el máximo de 36 en la escala de puntuación. El promedio para esta medición fue de 24,1 puntos.
El 75\% de los resúmenes evaluados obtuvo menos de 28 puntos en esta dimensión, el $50 \%$ menos de 24 puntos, y el $25 \%$ menos de 22.

Esta tabla señala que el 50\% de los cuerpos de los resúmenes están bajo la media, lo que está indicando que deben mejorarse los diversos ítemes presentados con baja frecuencia en la tabla anterior.

\section{Tabla 13}

De acuerdo a los resultados expuestos en la tabla, existe una relación altamente significativa $(\mathrm{p}=0,003)$ que apoya la hipótesis planteada: "A años mas recientes de ingreso al programa de magíster mayor puntaje de evaluación de la calidad global de los resúmenes de tesis". Hallazgo similar al estudio de Oria et al. (22), quienes concluyeron que la calidad de los resúmenes de tesis aumentaba a través de los años. Estos resultados pueden estar relacionados con la trayectoria presentada por el programa y la mayor experiencia investigativa que los docentes tutores de tesis presentan en el proceso de guiar a los tesistas del programa de maestría por los senderos del proceso de la investigación científica, y la elaboración del informe final, la tesis.

Al correlacionar cada dimensión de la pauta de evaluación aplicada con el año de ingreso del autor al programa, resulta una relación estadísticamente significativa en cada caso, por tanto en este estudio son apoyadas las hipótesis planteadas.

\section{Tabla 14}

El mundo académico está más relacionado -por las características intrínsecas del quehacer- con el proceso investigativo que el asistencial. Diversos autores $(32,17)$ señalan que las producciones científicas en el ámbito de la enfermería provienen mayoritariamente de enfermeras docentes. Al comparar los puntajes obtenidos por autores académicos y asistenciales, en este estudio se rechaza la hipótesis propuesta que señalaba que "los autores académicos presentan mejores pun- 
tajes en la evaluación de la calidad global de los resúmenes de tesis que los autores asistenciales" $(\mathrm{p}=0,07)$.

Al comparar la actividad del autor y cada dimensión de la pauta de evaluación, tampoco resulta una relación estadísticamente significativa en cada caso, por lo que no son apoyadas las hipótesis planteadas.

\section{CONCLUSIONES}

Este estudio da cuenta que, a medida que el ingreso de los alumnos al programa de magíster en enfermería de la universidad en estudio sea más reciente, éste se relaciona con el aumento en la evaluación de la calidad de los resúmenes de tesis, apoyando la hipótesis en este referente planteada para esta investigación. Esto representa un aspecto destacable para la institución y el programa de postgrado que lo imparte. Sin embargo, existen aspectos que deben ser mejorados, entre los que destaca lo relacionado con la presentación de las conclusiones, que no siempre tienen relación con los resultados y objetivos de la investigación. También debe mejorarse la presentación de los descriptores y la identificación completa de los respectivos autores; tanto del profesor guía como del alumno del programa.

Los resultados estadísticos entre la relación de la calidad de los resúmenes de las tesis y la actividad del autor (académico o asistencial) no demostraron diferencias significativas.

En cuanto a los fenómenos estudiados, existe una gran dispersión de éstos.

\section{Sugerencias}

En el contexto de los hallazgos obtenidos, las autoras consideran necesario continuar con las evaluaciones periódicas de la producción científica de los programas de postgrado dentro de las universidades, para detectar las deficiencias e implementar intervenciones orientadas a mejorar la calidad de la producción del postgrado. Estas evaluaciones debieran ser actividades rutinarias, dependiendo de la producción (al menos cada cinco años), realizadas bajo normativa de los programas y que se traduzcan en la optimización de la calidad de las tesis.

\section{TABLAS}

Tabla 1. Distribución según sexo y actividad de los autores.

\begin{tabular}{|l|l|c|c|}
\hline \multicolumn{2}{|c|}{} & $f$ & $\%$ \\
\hline \multirow{3}{*}{ Sexo } & Femenino & 79 & 94,0 \\
\cline { 2 - 4 } & Masculino & 5 & 6 \\
\hline \multirow{3}{*}{ Actividad } & Asistencial & 12 & 14,3 \\
\cline { 2 - 4 } & Académico & 72 & 85,7 \\
\hline
\end{tabular}


Tabla 2. Distribución de los autores según años en el programa.

\begin{tabular}{|c|c|c|}
\hline Años de Estudio & $f$ & $\%$ \\
\hline 2 & 6 & 7,1 \\
\hline 3 & 23 & 27,4 \\
\hline 4 & 12 & 14,3 \\
\hline 5 & 14 & 16,7 \\
\hline 6 & 20 & 23,8 \\
\hline 7 o más & 9 & 10,7 \\
\hline Total & 84 & 100 \\
\hline
\end{tabular}

Tabla 3. Distribución según fenómenos de estudios.

\begin{tabular}{|l|c|c|}
\hline Fenómenos de Estudio & $f$ & $\%$ \\
\hline Estilo de vida promotor / autocuidado & 10 & 11,9 \\
\hline Estrés / Burnout & 7 & 8,3 \\
\hline Percepción de violencia & 4 & 4,8 \\
\hline Calidad de vida & 4 & 4,8 \\
\hline Satisfacción laboral & 3 & 3,6 \\
\hline Sexualidad & 4 & 4,8 \\
\hline Adherencia control & 2 & 2,4 \\
\hline Tabaquismo & 4 & 4,8 \\
\hline Estrategias educativas & 2 & 2,4 \\
\hline Actitud hacia el envejecimiento & 2 & 2,4 \\
\hline Funcionalidad del anciano & 2 & 2,4 \\
\hline Muerte y proceso de morir & 4 & 4,8 \\
\hline Otros fenómenos & 36 & 42,9 \\
\hline Total & 84 & 100,0 \\
\hline
\end{tabular}

Tabla 4. Distribución de sujetos de estudio según condición de salud.

\begin{tabular}{|c|c|c|c|c|}
\hline Sujetos de estudio & \multicolumn{2}{|l|}{ Condición } & $f$ & $\%$ \\
\hline \multirow[t]{3}{*}{ Individuo } & \multicolumn{2}{|l|}{ Con Patologías } & 18 & 21,4 \\
\hline & \multirow[t]{2}{*}{ Sin Patologías } & Enfermeras & 25 & 29,8 \\
\hline & & Otros & 32 & 38,1 \\
\hline \multirow{2}{*}{ Familia } & \multicolumn{2}{|c|}{ Con integrantes afectados por una patología } & 5 & 6 \\
\hline & \multicolumn{2}{|c|}{ Con integrantes sanos } & 3 & 3,6 \\
\hline Comunidad & \multicolumn{2}{|l|}{ Sana } & 1 & 1,2 \\
\hline \multicolumn{3}{|l|}{ Total } & 84 & 100 \\
\hline
\end{tabular}


Tabla 5. Distribución según Abordaje Metodológico.

\begin{tabular}{|l|l|c|c|}
\hline Abordaje Metodológico & \multicolumn{1}{|c|}{ Diseño / Estrategia } & $f$ & $\%$ \\
\hline \multirow{4}{*}{ Cuantitativo } & Descriptivo & 2 & 2,4 \\
\cline { 2 - 4 } & Correlacional & 68 & 81,0 \\
\cline { 2 - 4 } & Experimental & 8 & 9,5 \\
\hline \multirow{2}{*}{ Cualitativo } & Fenomenológico & 4 & 4,8 \\
\cline { 2 - 4 } & Interaccionismo Simbólico & 1 & 1,2 \\
\hline \multicolumn{2}{|l}{ Total } & 84 & 100,0 \\
\hline
\end{tabular}

Tabla 6. Estadísticos descriptivos de puntajes obtenidos en la medición de la calidad global de los resúmenes de tesis de magíster.

\begin{tabular}{|c|c|c|c|c|c|c|c|c|c|c|c|}
\hline \multirow{2}{*}{$\mathrm{N}$} & \multirow{2}{*}{ Media } & \multirow{2}{*}{ Mediana } & \multirow{2}{*}{ Moda } & \multirow{2}{*}{$\begin{array}{c}\text { Std. } \\
\text { Desviación }\end{array}$} & \multirow{2}{*}{ Varianza } & \multirow{2}{*}{ Rango } & \multirow{2}{*}{ Mínimo } & \multirow{2}{*}{ Máximo } & \multicolumn{3}{|c|}{ Percentiles } \\
\hline & & & & & & & & & 25 & 50 & 42 \\
\hline 84 & 37,8929 & 38 & 36 & 5,68239 & 32,29 & 34 & 14 & 48 & 36 & 38 & 42 \\
\hline
\end{tabular}

Tabla 7. Distribución de los ítemes que componen la dimensión de títulos de los resúmenes de las tesis del programa de Magíster en Enfermería.

\begin{tabular}{|l|c|c|c|c|c|c|}
\hline \multirow{2}{*}{ Ítemes del título } & \multicolumn{2}{|c|}{ Sí } & \multicolumn{2}{|c|}{ Dudoso } & \multicolumn{2}{c|}{ No } \\
\cline { 2 - 7 } & $f$ & $\%$ & $f$ & $\%$ & $f$ & $\%$ \\
\hline Es indicativo del estudio & 78 & 92,9 & 5 & 6 & 1 & 1,2 \\
\hline Es claro, fácil de entender & 83 & 98,8 & 1 & 1,2 & 0 & 0 \\
\hline Es conciso (máx 15 palabras) & 52 & 61,9 & 17 & 20,2 & 15 & 17,9 \\
\hline Utiliza palabras completas & 80 & 95,2 & 1 & 1,2 & 3 & 3,6 \\
\hline Usa tono afirmativo & 83 & 98,8 & 1 & 1,2 & 0 & 0 \\
\hline Es gramaticalmente correcto & 81 & 96,4 & 3 & 3,6 & 0 & 0 \\
\hline
\end{tabular}

Tabla 8. Estadísticos descriptivos de puntajes obtenidos en la evaluación de la calidad del título de los resúmenes de tesis de magíster.

\begin{tabular}{|c|c|c|c|c|c|c|c|c|c|c|c|}
\hline N & Media & Mediana & Moda & $\begin{array}{c}\text { Std. } \\
\text { Desviación }\end{array}$ & Varianza & Rango & Mínimo & Máximo & \multicolumn{4}{|c|}{ Percentiles } \\
\cline { 5 - 10 } & & & & & & & & 25 & 50 & 42 \\
\hline
\end{tabular}


Tabla 9. Distribución de los ítemes que componen la dimensión de Evaluación de autores de los resúmenes de las tesis del programa de Magíster en Enfermería.

\begin{tabular}{|l|c|c|c|c|c|c|}
\hline \multirow{2}{*}{ Itemes respecto a autores } & \multicolumn{2}{|c|}{ Sí } & \multicolumn{2}{|c|}{ Dudoso } & \multicolumn{2}{c|}{ No } \\
\cline { 2 - 7 } & $f$ & $\%$ & $f$ & $\%$ & $f$ & $\%$ \\
\hline Identificación Alumno y Prof. Guía & 39 & 46,4 & 45 & 53,6 & 0 & 0 \\
\cline { 2 - 7 } Usa nombres completos & 36 & 42,9 & 48 & 57,1 & 0 & 0 \\
\hline
\end{tabular}

Tabla 10. Estadísticos descriptivos de puntajes obtenidos en la evaluación de la calidad de los autores de los resúmenes de tesis de magíster.

\begin{tabular}{|c|c|c|c|c|c|c|c|c|c|c|c|}
\hline N & Media & Mediana & Moda & Desviación & Varianza & Rango & Mínimo & Máximo & \multicolumn{4}{|c|}{ Percentiles } \\
\cline { 7 - 11 } & & & & & & & & 25 & 50 & 42 \\
\hline 84 & 2,8929 & 2,0000 & 2,00 &, 95713 &, 916 & 2,00 & 2,00 & 4,00 & 2,0000 & 2,0000 & 4,0000 \\
\hline
\end{tabular}

Tabla 11. Distribución de los ítemes que componen la dimensión cuerpo de resúmenes de tesis de programa de Magíster en Enfermería.

\begin{tabular}{|c|c|c|c|c|c|c|}
\hline \multirow[t]{2}{*}{ Itemes Cuerpo Resumen } & \multicolumn{2}{|c|}{ Sí } & \multicolumn{2}{|c|}{ Dudoso } & \multicolumn{2}{|c|}{ No } \\
\hline & $f$ & $\%$ & $f$ & $\%$ & $f$ & $\%$ \\
\hline $\begin{array}{l}\text { Permite identificar el contenido en forma } \\
\text { rápida y exacta }\end{array}$ & 76 & 90.5 & 4 & 4.8 & 4 & 4.8 \\
\hline Es claro, fácil de entender & 75 & 89.3 & 4 & 4.8 & 5 & 6.0 \\
\hline Describe Objetivos / Hipótesis & 80 & 95.2 & 1 & 1.2 & 3 & 3.6 \\
\hline Describe el diseño & 68 & 81.0 & 7 & 8.3 & 9 & 10.7 \\
\hline Describe los resultados & 60 & 71.4 & 12 & 14.3 & 12 & 14.3 \\
\hline $\begin{array}{l}\left(^{*}\right) \text { Describe conclusión y/o consideracio- } \\
\text { nes relacionadas con resultados }\end{array}$ & 11 & 13,1 & 2 & 2,4 & 71 & 84,5 \\
\hline $\begin{array}{l}\left.{ }^{\star}\right) \text { Describe conclusiones y/o } \\
\text { consideraciones finales relacionadas con } \\
\text { objetivos }\end{array}$ & 15 & 17,9 & 4 & 4,8 & 65 & 77,4 \\
\hline Es conciso (máx. 300 palabras) & 69 & 82,1 & 2 & 2,4 & 13 & 15,5 \\
\hline $\begin{array}{l}\left.{ }^{*}\right) \text { Presenta resultados numéricos } \\
\text { (cuantitativo) o juicios (cualitativas) }\end{array}$ & 36 & 42,9 & 2 & 2,4 & 46 & 54,8 \\
\hline Usa palabras completas & 80 & 95,2 & 0 & 0 & 4 & 4,8 \\
\hline Usa solamente texto & 82 & 97,6 & 0 & 0 & 2 & 2,4 \\
\hline $\begin{array}{l}\text { En el texto no cita referencias } \\
\text { bibliográficas }\end{array}$ & 81 & 96,4 & 0 & 0 & 3 & 3,6 \\
\hline $\begin{array}{l}\left({ }^{*}\right) \text { Presenta descriptores congruentes con } \\
\text { el título }\end{array}$ & 30 & 35,7 & 4 & 4,8 & 50 & 59,5 \\
\hline
\end{tabular}

$\left.{ }^{*}\right)$ Itemes con menor frecuencia. 
Tabla 12. Estadísticos descriptivos de puntajes obtenidos en la evaluación de la calidad del cuerpo de los resúmenes de tesis de magíster.

\begin{tabular}{|c|c|c|c|c|c|c|c|c|c|c|c|}
\hline N & Media & Mediana & Moda & $\begin{array}{c}\text { Std. } \\
\text { Desviación }\end{array}$ & Varianza & Rango & Mínimo & Máximo & \multicolumn{4}{|c|}{ Percentiles } \\
\cline { 5 - 10 } & & & & & & & 25 & 50 & 75 \\
\hline
\end{tabular}

Tabla 13. Correlación entre año de ingreso al programa de magíster y puntajes globales y por dimensiones de los resúmenes.

\begin{tabular}{|l|c|c|}
\hline Dimensión & $\begin{array}{c}\text { Correlación Pearson } \\
\text { (Año de Ingreso/Puntajes) }\end{array}$ & $\mathrm{p}$ \\
\hline Global &, 294 & 0,003 \\
\hline Título &, 198 & 0,036 \\
\hline Autores &, 488 & 0 \\
\hline Cuerpo del resumen &, 218 & 0,023 \\
\hline
\end{tabular}

Tabla 14. Comparación entre las actividades de los autores y los puntajes obtenidos en las diversas dimensiones.

\begin{tabular}{|l|c|c|c|}
\hline Dimensión & $\begin{array}{c}\mathrm{t} \\
\text { Entre actividades } \\
\text { autor }\end{array}$ & Sign (2 tailed) & Sign (1 tailed) \\
\hline Global & 1,421 & 0,159 & 0,07 \\
\hline Título & 0,219 & 0,827 & 0,41 \\
\hline Autores & $-0,123$ & 0,902 & 0,45 \\
\hline Cuerpo del resumen & 1,218 & 0,227 & 0,11 \\
\hline
\end{tabular}

$\left.{ }^{*}\right) \mathrm{p}<0.05$

\section{REFERENCIAS}

1. Sanz L. Evaluación de la investigación y sistema de ciencia. Documento de Trabajo Consejo Superior de Investigaciones Científicas 2004. Extraído el 20 de julio 2008. Disponible en: http://www. iesam.csic.es/doctrab2/dt-0407.pdf

2. Rosell J, Bobenrieth M. Metodología de la escritura científica en clínica. Revista Andaluza de Patología Digestiva 2000; 23 (4); 155-161.

3. Bobenrieth M. Normas para revisión de artículos originales en Ciencias de la Salud. Revista Internacional Psicología Clínica y de la Salud 2002; 2 (3): 509-523.

4. Bolseguí M, Fuguet A. Cultura de la evaluación: Una aproximación conceptual. Revista Investigación y Postgrado 2006; 


\section{1 (1): 77-78.}

5. Fairman J. Context and contingency in the history of post World war II nursing scholarship in the United States. Journal Nursing Scholarship 2008; 40 (1): 4-10.

6. Carvalho V. Acerca de las bases teóricas, filosóficas, epistemológicas de la investigación científica, el caso de la enfermería. Rev. Latino-Am Enfermagem 2003. Vol 11(6): 807-815.

7. Palucci M, Costa I. La Inserción social del postgrado en enfermería. Rev. LatinoAm Enfermagem 2007; 15(5):887-8. Extraído el 10 de junio 2008. Disponible en: www.eerp.usp.br/rlae

8. Ferrando G. Evaluación de la calidad de la educación continua. Postgrados y postítulos. Calidad en la Educación 2003; 1-10.

9. Johnson S H. Adapting a thesis to publication style: meeting editors' expectations. Dimens Crit Care Nurs. 1997; 15 (3): 160-167.

10. Rosas A, Flores D, Valarino E. Rol del tutor de tesis: Competencias, condiciones personales y funciones. Investigación y Postgrado 2006; 21 (1): 153-185.

11. Valarino E, Meneses R, Yaber G, Pujol L. Veinte años de investigación en los trabajos de grado de maestría en psicología de la Universidad Simón Bolívar. Investigación y Postgrado 2001; 16(2): 73-102.

12. Barbosa A. Diez años de investigación ornitológica en España a través de las tesis doctorales 2000. Extraído el 18 de julio 2008. Disponible en: http://www. ardeola.org/files/ardeola_459.pdf

13. Camps D, Recuero Y, Samar M, Ávila R. Análisis bibliométrico de tesis de doctorado del área de las ciencias de la salud: primera parte, odontología. Rev. Fac. Cienc. Med. 2005; 62(3):53-56.

14. Calani F, Chuquimia W. Características discursivas, organizativas y metodológicas de las tesis de grado. Clínico 2003; 48(1):7-12.

15. Valarino E. Rol del tutor de tesis: Com- petencias, condiciones personales y funciones Investigación y Postgrado 2000; 21(1):153-185.

16. Sanabria H, Bullón L. Exploración de la calidad de las tesis de enfermería de la Facultad de Medicina de San Fernando. Universidades 2000; 20: 5-10.

17. Alarcón A, Astudillo P. La investigación en enfermería en revistas latinoamericanas Cienc. enferm., 2000; 13 (2): 25-31.

18. Almeida M, Silva E, Villa T, Assis M, Kemura M. O conhecimento produzido no programa de mestrado de enfermagem em saúde pública da Escola de Enfermagem de Ribeirão Preto-USP e suas relações com as práticas de saúde. Rev. Latino-Am. Enfermagem 2000 [online]; 8 (5): 91-96. Extraído el 20 de junio 2008. Disponible en: http://www.scielo.br/pdf/ rlae/v8n5/12373.pdf

19. Egry E, Fonseca R, Bertolozzi M, Oliveira Ma, Takahashi R. Construindo o conhecimento em saúde coletiva: uma análise das teses e dissertações produzidas. Rev Esc Enferm. USP 2005.39: 544-552.

20. Ferreira E, Santana E, Oliveira M. Producción científica en salud de la mujer en el postgrado de enfermería de la Universidad Federal de Ceará, Brasil 1993-2002. Cienc. enferm., 2005; 11 (2): 59-70.

21 Erdmann A, Silva I, Rodrigues R, Fernandes J, Vianna L, Lopes M, Santos R. et al. Teses produzidas nos programas de Pós-Graduação em Enfermagem de 1983 a 2001 Rev Esc Enferm. USP 2005; 39:497-505.

22. Backes D, Macêdo F, Schaefer A. Concepções de cuidado: uma análise das teses apresentadas para um programa de pós-graduação em enfermagem. Texto contexto - enfermeria. 2006. Extraído el 15 de julio 2008. Disponible en: <http:// www.scielo.br/scielo.php?scrip

23. Oria M, Moraes L, Vale E. Análise crítica dos resumos de teses de um Programa de Pós-Graduação. Acta paul. enferm. 2007; 20 (2): 186-190. 
24. Mendoza S. Producción científica de la enfermería latinoamericana, período 1959-2005. Tesis optar grado Doctora en Enfermería. Universidad de Concepción, 2006. Chile.

25. Carlino P. ¿Por qué no se completan las tesis en los postgrados?: Obstáculos percibidos por maestrandos en curso y magistri exitosos. La Revista Venezolana de Educación (Educere) 2005; 30: 415-420.

26. Estebánez M. La participación de las mujeres en el sistema de ciencia y tecnología Argentino. Proyecto GENTEC, Informe Final 2003. Extraído 20 de mayo 2008. Disponible desde: http: //www.centroredes.org.ar

27. Velandia A. Areas y líneas, grupos y centros de investigación en el desarrollo del conocimiento de enfermería. Cienc. enferm., 1997; III (1): 13-22.

28 Mendoza S, Paravic T. Origen, clasificación y desafíos de las revistas científicas. Investigación y Postgrado 2006; 21 (1): 49-76.
29. Amezcua M, Gálvez A. Los modos de análisis en investigación cualitativa en salud: perspectiva crítica y reflexiones en voz alta. Rev. Esp. Salud Pública 2002; 76:423-436.

30. Arteaga O. Investigación en salud y métodos cualitativos. Revista ciencia y trabajo 2006; (21):151-153. Extraído el 18 de abril 2008. Disponible en: www. cienciaytrabajo.cl/pdfs/21/pagina $\% 20$ 151.pdf

31. Conde F. Encuentros y desencuentros entre la perspectiva cualitativa y la cuantitativa en la historia de la medicina. Rev. Esp. Salud Pública 2002; (76): 395-408.

32. Paravic T, Mendoza S. Producción científica en enfermería: tendencia y calidad de las comunicaciones del IV Coloquio Panamericano de investigación en enfermería Concepción-Chile. Cienc. enferm., 1996; II (1): 51-60. 